\title{
Avaliação do tratamento dos distúrbios vestibulares na criança através da posturografia dinâmica computadorizada: resultados preliminares
}

\author{
Evaluation of the treatment of vestibular disorders in children \\ with computerized dynamic posturography: preliminary results \\ Ítalo R.T. Medeiros ${ }^{1}$, Roseli S.M. Bittar ${ }^{2}$, Maria Elisabete B. Pedalini ${ }^{3}$, \\ Maria Cecília Lorenzi ${ }^{4}$, Márcia A. Kii ${ }^{1}$, Lázaro G. Formigoni ${ }^{5}$
}

\section{Resumo}

Objetivo: o objetivo desta investigação foi avaliar a posturografia como método de acompanhamento de crianças com vestibulopatia periférica, tratadas com reabilitação vestibular, estabelecendo sua correlação com a evolução clínica dos pacientes.

Métodos: dez crianças (seis meninos e quatro meninas) portadoras de afecções vestibulares periféricas, submetidas à reabilitação vestibular como forma de tratamento, tiveram sua evolução clínica avaliada através de uma anamnese detalhada de seus sintomas e da realização da posturografia dinâmica computadorizada. Os dados posturográficos foram analisados e comparados à evolução clínica dos pacientes estudados.

Resultados: observou-se, após o tratamento, melhora significativa das condições 1 (paciente em posição ortostática, plataforma fixa e olhos abertos) e 5 (paciente em posição ortostática, plataforma em movimento e olhos fechados) da posturografia dinâmica, da função vestibular e do índice do equilíbrio, que correlacionaram-se significativamente com a melhora clínica e diminuição dos sintomas dos pacientes. Observou-se, também, redução significativa da influência da função somatosensorial sobre o equilíbrio final da criança.

Conclusão: os dados obtidos mostram que a posturografia não substitui a avaliação clínica convencional, mas agrega dados quantitativos importantes para o acompanhamento da terapia destes pacientes.

J Pediatr (Rio J) 2003;79(4):337-42: criança, tontura, doenças vestibulares, testes de função vestibular, reabilitação.

\begin{abstract}
Objective: this study aimed at evaluating dynamic posturography as an evaluation method in children with balance problems due to peripheral vestibulopathy, before and after treatment with vestibular rehabilitation, establishing its correlation with classical clinical evaluation.

Method: ten children (six boys and four girls) with vestibular symptoms of peripheral origin were evaluated through a complete clinical history and with dynamic computerized posturography after being treated by vestibular rehabilitation therapy. Posturographic data were analyzed and compared to standard clinical evaluation parameters.
\end{abstract}

Results: dynamic posturography showed a significant improvement of condition 1 (orthostatic position, fixed support and open eyes) and 5 (orthostatic position, sway-referenced support and closed eyes) of the vestibular function and of the composite balance score. The data showed significant correlation with the clinical improvement observed. A significant reduction of proprioceptive influence was also observed.

Conclusion: data showed that the dynamic posturography adds important quantitative information to the conventional clinical evaluation of vestibular symptoms, especially in children.

J Pediatr (Rio J) 2003;79(4):337-42: child, dizziness, vestibular diseases, vestibular function tests, rehabilitation.

1. Doutor em Otorrinolaringologia pela Faculdade de Medicina da Universidade de São Paulo (FMUSP). Médico colaborador do setor de Otoneurologia do Hospital das Clínicas da FMUSP (HCFMUSP).

2. Doutora em Otorrinolaringologia pela FMUSP. Médica do setor de Otoneurologia do HCFMUSP.

3. Fonoaudióloga responsável pelo ambulatório de reabilitação vestibular da FMUSP. Pós-graduanda, nível Mestrado - FMUSP.

4. Doutora em Otorrinolaringologia pela FMUSP.

5. Professor Associado da FMUSP.

Fonte financiadora: FAPESP.

Artigo submetido em 18.11.02, aceito em 12.03.03. 


\section{Introdução}

A tontura é um sintoma muitas vezes esquecido ou desprezado na anamnese da criança. Essa queixa pode associar-se a várias outras afecções, muitas vezes diferindo daquelas encontradas em adultos. É em função dessa dificuldade que pediatras, neurologistas e até otorrinolaringologistas não estão atentos para a presença de quadros vestibulares na infância.

As repercussões sociais e emocionais dessas queixas nesta faixa etária (infância) são muito mais deletérias do que em adultos ${ }^{1}$. O afastamento ou isolamento afetivo (introspeção), os distúrbios escolares, as alterações no sono e as fobias trazem comprometimento importante do desenvolvimento. As situações que envolvem movimentos ressaltam a insegurança gerada pela vestibulopatia, de modo que atividades normais da infância, como andar de bicicleta, brincar em parques, participar de jogos infantis e esportes passam a ser evitados ${ }^{2}$.

Uma vez definido o diagnóstico de vestibulopatia na criança, deve-se instituir a forma mais adequada de tratamento. A conduta pode variar desde expectante, nos casos mais leves e sem repercussões clínicas, ou sociais, até a utilização de drogas sedativas labirínticas ${ }^{2}$. Os medicamentos utilizados na criança são basicamente os mesmos empregados em adultos, e trazem consigo os correspondentes efeitos colaterais. $O$ tratamento do equilíbrio corporal através da reabilitação vestibular vem sendo por nós empregado em crianças com sucesso ${ }^{3}$.

O maior problema encontrado nesta faixa etária é a dificuldade de quantificação objetiva de seus sintomas. $\mathrm{Na}$ maioria das vezes, os pequenos pacientes caracterizam com dificuldade suas queixas, de modo que geralmente seus responsáveis são os verdadeiros informantes, trazendo os dados mais importantes da anamnese. O mesmo acontece com relação à caracterização da resposta ao tratamento instituído. Nesse contexto, a posturografia dinâmica computadorizada (PDC) surge como veículo útil na quantificação dessa sintomatologia, antes e depois do tratamento, qualquer que seja a terapêutica proposta ${ }^{4}$.

A PDC é um exame utilizado para avaliação geral do equilíbrio que integra as informações labirínticas, visuais e somatosensoriais. O teste mais utilizado, denominado "teste de organização sensorial" (SOT - sensory organization test), é composto por seis condições sensoriais, cujas respostas são percebidas e registradas através de uma plataforma móvel (informações somatosensoriais), a presença ou ausência da visão (informações visuais) e as aferências vestibulares.

Dada a subjetividade da avaliação clínica nos casos de vestibulopatia na infância e o surgimento da PDC como exame que possibilita a quantificação das condições envolvidas no equilíbrio, foi concebido este estudo com a principal finalidade de analisar dados posturográficos anteriores e posteriores ao tratamento de crianças vestibulopatas, através da reabilitação vestibular, comparando estes resul- tados com os dados clínicos obtidos, e determinando o papel desta nova opção diagnóstica nestes casos.

\section{Material e métodos}

Foram avaliadas, prospectivamente, através de um estudo observacional, dez crianças consecutivamente atendidas no Ambulatório de Vertigem na Infância do Hospital das Clínicas da FMUSP (HCFMUSP), com queixa de tontura, associada ou não à cefaléia, originárias do ambulatório de neuropediatria ou otorrinolaringologia geral deste mesmo hospital.

Constituíram-se critérios de exclusão do estudo, presença de alteração funcional do ouvido médio, doenças neurológicas degenerativas e problemas vestibulares de origem metabólica.

Após prévia autorização da Comissão de Ética em pesquisa do HCFMUSP, os pais ou responsáveis pela criança foram solicitados a assinar termo de consentimento assistido após esclarecimento e concordância em participar do estudo (protocolo HC - 187/98). As crianças, então, foram submetidas à anamnese detalhada conjunta (passiva e ativa) com seus acompanhantes. O diagnóstico da vestibulopatia foi firmado através de exame eletronistagmográfico, incluindo em alguns casos, a prova em cadeira rotatória (PRPD), para reforço do diagnóstico. Foi também realizada avaliação audiológica completa, para afastar doenças de ouvido médio, cuja presença constituía critério de exclusão.

Além da avaliação neurológica, foram realizados, em todas as crianças, exames metabólicos (glicemia de jejum, colesterol total e frações, triglicérides e hormônios tireoideanos) e reações sorológicas para a sífilis. A presença de alterações de qualquer uma destas variáveis também tornava a criança inelegível para o protocolo.

A seguir, as crianças portadoras de vestibulopatias foram submetidas à PDC com o aparelho Equitest System ${ }^{\circledR}$ - Versão 4.0, produzido pela NeuroCom International ${ }^{\circledR}$ _ USA.

Na posturografia convencional (Equitest ${ }^{\circledR}$ ), são avaliadas seis condições no teste de organização sensorial:

- condição 1: paciente em posição ortostática, plataforma fixa e olhos abertos;

- condição 2: paciente em posição ortostática, plataforma fixa e olhos fechados (Romberg clássico);

- condição 3: paciente em posição ortostática, plataforma fixa, olhos abertos e visão referenciada pelo movimento;

- condição 4: paciente em posição ortostática, plataforma em movimento e olhos abertos;

- condição 5: paciente em posição ortostática, plataforma em movimento e olhos fechados;

- condição 6: paciente em posição ortostática, plataforma e visão em movimento, com olhos abertos. 
A meta em cada uma destas condições é a manutenção do equilíbrio estático. O paciente é orientado a permanecer o mais imóvel possível na plataforma, mesmo diante das oscilações da mesma e do campo visual envolvente. A quantificação dos resultados obtidos varia de $100 \%$ (não houve deslocamento algum registrados pelos sensores da plataforma) até $0 \%$, que corresponde à queda em qualquer uma das direções. Baseado nos dados encontrados nestas condições, o Equitest ${ }^{\circledR}$ é capaz de calcular a média de cada uma delas, um índice de análise da função somatosensorial, visual, vestibular e a relação entre a informação visual e vestibular, denominada preferência visual, além de um índice de equilíbrio, que são descritas a seguir:

- função somatosensorial: média da condição 2 / média da condição 1;

- função visual: média da condição 4 / média da condição 1;

- função vestibular: média da condição 5 / média da condição 1;

- preferência visual: média da condição $3+6 /$ média da condição $2+5$

- indice de equilíbrio: média aritmética das três repetições da condição 1 e 2 e os dois melhores resultados da condições $3,4,5$ e 6 .

Realizada a primeira posturografia em todas as crianças do estudo, foi então iniciado o programa de reabilitação vestibular (RV), com o auxílio dos familiares, consistindo de sessões de orientação e exercícios baseados na técnica descrita por Cawthorne \& Cooksey, modificada e adaptada para nossa população ${ }^{5}$. Os exercícios desempenhados baseiam-se numa série de movimentos repetidos de cabeça, pescoço e olhos que visam a estimular o processo de compensação do labirinto. Em nenhuma criança, mesmo nas menores, precisamos adaptar exercícios diferentes dos utilizados para adultos. Quatro sessões de acompanhamen- to foram realizadas $\left(1^{\circ}, 15^{\circ}, 30^{\circ} \text { e } 60^{\circ} \text { dias }\right)^{3,6}$. Ao final da terapia de reabilitação vestibular, realizada sempre pela mesma fonoaudiológa, os pacientes foram submetidos a novo exame pela PDC.

A metodologia de análise incluiu ferramentas da estatística descritiva e da estatística não-paramétrica. $\mathrm{Na}$ avaliação clínica do resultado terapêutico da $\mathrm{RV}$, o número de sintomas presentes antes e depois do tratamento e sua variação constituíram variáveis de análise. Além disso, observou-se a ocorrência de cura, melhora ou de quadros inalterados após o tratamento. A presença de cinetose também foi observada.

O teste de Wilcoxon foi empregado na avaliação dos vários resultados anteriores e posteriores ao tratamento. $\mathrm{O}$ percentual de melhora da condição $n^{\circ} 5$ (cinco) foi calculado segundo a equação: [(valor final - valor inicial) $\div$ valor inicial] x 100.

A correlação entre a avaliação clínica subjetiva e o resultado da avaliação pela PDC (percentual de melhora da condição 5) foi estudada com o teste de Spearman.

O nível de significância considerado foi de 0,05 , como preconizado em ensaios biológicos $(\mathrm{p}<0,05)$.

\section{Resultados}

Dos dez pacientes avaliados, seis eram do sexo masculino, e quatro do sexo feminino. A idade variou entre 5,4 anos e 10,3 anos (média $=8,3 \pm 1,6$ ano).

\section{Avaliação clínica do resultado da terapêutica institu- ída}

Os dados obtidos para os dez pacientes estudados podem ser observados na Tabela 1.

Tabela 1 - Sintomas observados nas dez crianças vestibulopatas estudadas

\begin{tabular}{|c|c|c|c|c|c|c|c|c|c|c|}
\hline & Tontura & Cefaléia & Cinetose & $\begin{array}{c}\text { Náuseas } \\
\text { e } \\
\text { vômitos }\end{array}$ & Palidez & $\begin{array}{c}\text { Dor } \\
\text { abdominal }\end{array}$ & $\begin{array}{l}\text { Problemas } \\
\text { com } \\
\text { brinquedos }\end{array}$ & Quedas & $\begin{array}{l}\text { Dist. } \\
\text { sono }\end{array}$ & $\begin{array}{l}\text { Desvio } \\
\text { cabeça }\end{array}$ \\
\hline Paciente 1 & + & + & + & + & + & + & + & & & \\
\hline Paciente 2 & + & + & & & & + & + & + & + & \\
\hline Paciente 3 & + & + & & & & & + & & & + \\
\hline Paciente 4 & + & + & & + & & & + & & & \\
\hline Paciente 5 & + & + & & & & & & & & \\
\hline Paciente 6 & + & & + & + & & & + & & & \\
\hline Paciente 7 & + & + & & + & & & + & & & \\
\hline Paciente 8 & + & + & + & + & & & & + & & \\
\hline Paciente 9 & + & + & + & + & & & & & & \\
\hline Paciente 10 & + & & + & + & & & & & & \\
\hline Total & 10 & 8 & 5 & 7 & 1 & 2 & 6 & 2 & 1 & 1 \\
\hline
\end{tabular}


Todos os pacientes referiram tontura na anamnese. Outros sintomas clínicos freqüentes incluíram cefaléia (80\%), náuseas e vômitos (70\%), problemas com brinquedos $(60 \%)$ e cinetose $(50 \%)$ dos casos. Também foram observados dores abdominais, tendência a quedas (em 20\% dos casos), palidez e desvio de segmento cefálico (em 10\% dos casos).

Um quadro geral com o número de sintomas iniciais e finais (após o tratamento), bem como a categorização da evolução clínica de cada caso, podem ser vistos na Tabela 2. A cinetose relatada por algumas crianças apresentou melhora clínica com redução na intensidade de tal sintoma, sem, contudo, haver a remissão total da mesma. Observamos, também, que em nenhum caso o quadro clínico permaneceu inalterado após a terapêutica (40\% de melhora e $60 \%$ de remissão dos sintomas clínicos).

\section{Avaliação através da PDC dos mesmos pacientes}

A comparação das médias das várias condições estudadas pela posturografia dinâmica antes e depois do tratamento por RV pode ser observada na Tabela 3 .

Observamos um aumento significativo dos valores numéricos das condições 1 e 5 . A condição 1 corresponde à posição ortostática de olho aberto, e a condição 5 à posição ortostática de olhos fechados com uma plataforma móvel. Esta última representa a melhor condição para avaliação do sistema vestibular, visto que as informações visuais e proprioceptivas são desprezíveis. Observamos também uma redução significativa da função somatosensorial e limítrofe da condição 2 .

O percentual médio de melhora da condição 5 foi de $29,0 \%$.

\section{Estudo da correlação entre avaliação clínica e avali-} ação pela PDC

A correlação entre a avaliação clínica antes e depois do tratamento (medida pela variação do número de sintomas presentes antes e depois do tratamento $-4^{\mathrm{a}}$ coluna da Tabela 2) e a variação do desempenho vestibular (medida pelo percentual de melhora da condição 5) mostrouse negativa e moderada $(r=-0,50)$, indicando que quanto mais importante a redução no número de sintomas, melhor o desempenho vestibular pós-tratamento e maior o valor da condição 5 .

Estudando-se separadamente os grupos de pacientes que apresentaram remissão da sintomatologia $(n=6)$ e aqueles que apresentaram apenas melhora parcial, mantendo uma leve cinetose após o tratamento ( $n=4)$, não observamos diferença significativa entre os valores da condição 5 inicial em cada grupo (desempenho vestibular inicial semelhante). Também não houve diferença entre o percentual de melhora do desempenho vestibular entre os dois grupos de pacientes, embora tenhamos observado uma tendência a um maior ganho de desempenho nos pacientes que apresentaram remissão final do quadro (Figura 1).

\section{Discussão}

Hubbell \& Skoner ${ }^{7}$, em sua revisão sobre vertigem na infância, citam a posturografia como método diagnóstico para tonturas na infância, mas não abordam mais profundamente o assunto. A ausência de dados sobre posturografia em crianças foi pontuada pelo consenso sobre métodos diagnósticos do sistema vestibular da Academia NorteAmericana de Neurologia ${ }^{4}$.

Tabela 2 - Variação no número de sintomas após o tratamento com RV e evolução clínicas das crianças estudadas

\begin{tabular}{ccccccc}
\hline Paciente & $\begin{array}{c}\mathbf{N}^{\mathbf{0}} \text { de sintomas } \\
\text { iniciais }\end{array}$ & $\begin{array}{c}\mathbf{N}^{\mathbf{0}} \text { de sintomas } \\
\text { após tratamento }\end{array}$ & $\begin{array}{c}\text { Variação } \\
\text { sintomas }\end{array}$ & $\begin{array}{c}\text { Evolução } \\
\text { clínica }\end{array}$ & $\begin{array}{c}\text { Cinetose antes } \\
\text { do tratamento }\end{array}$ & $\begin{array}{c}\text { Cinetose depois } \\
\text { do tratamento }\end{array}$ \\
\hline $\mathbf{1}$ & 7 & 0 & -7 & remissão & + & - \\
$\mathbf{2}$ & 6 & 0 & -6 & remissão & - & - \\
$\mathbf{3}$ & 4 & 0 & -4 & remissão & - & - \\
$\mathbf{4}$ & 4 & 0 & -4 & remissão & - & - \\
$\mathbf{5}$ & 2 & 0 & -2 & remissão & - & + \\
$\mathbf{6}$ & $\mathbf{4}$ & $\mathbf{1}$ & $\mathbf{- 3}$ & melhora & ++ & - \\
$\mathbf{7}$ & 4 & 0 & -4 & remissão & - & + \\
$\mathbf{8}$ & $\mathbf{5}$ & $\mathbf{1}$ & $\mathbf{- 4}$ & melhora & ++ & + \\
$\mathbf{9}$ & $\mathbf{4}$ & $\mathbf{1}$ & $\mathbf{- 3}$ & melhora & ++ & + \\
$\mathbf{1 0}$ & $\mathbf{3}$ & $\mathbf{1}$ & $\mathbf{- 2}$ & melhora & ++ & + \\
\hline
\end{tabular}


Tabela 3 - Médias numéricas das várias condições antes e depois do tratamento por RV

\begin{tabular}{|c|c|c|c|c|}
\hline & $\begin{array}{c}\text { Média antes } \\
\text { do tratamento } \\
( \pm \text { desvio padrão) }\end{array}$ & $\begin{array}{c}\text { Média depois } \\
\text { do tratamento } \\
( \pm \text { desvio padrão) }\end{array}$ & Análise estatística & \\
\hline Condição 1 & $89,7 \pm 3,3$ & $91,8 \pm 2,6$ & Aumento significativo & $(\mathbf{p}<\mathbf{0 , 0 1})$ \\
\hline Condição 2 & $89,0 \pm 2,8$ & $87,9 \pm 3,3$ & Redução não significativa & $(p=0,05)$ \\
\hline Condição 3 & $88,3 \pm 4,5$ & $88,7 \pm 4,0$ & Aumento não significativo & $(\mathrm{p}>0,05)$ \\
\hline Condição 4 & $69,0 \pm 7,9$ & $73,9 \pm 10,2$ & Aumento não significativo & $(\mathrm{p}>0,05)$ \\
\hline Condição 5 & $47,7 \pm 11,8$ & $59,5 \pm 10,0$ & Aumento significativo & $(\mathrm{p}<\mathbf{0 , 0 1})$ \\
\hline Condição 6 & $51,0 \pm 15,6$ & $56,4 \pm 6,5$ & Aumento não significativo & $(\mathrm{p}>0,05)$ \\
\hline Somatosensorial & $99,3 \pm 2,5$ & $95,8 \pm 2,5$ & Redução significativa & $(\mathbf{p}=\mathbf{0 , 0 1})$ \\
\hline Sistema visual & $77,0 \pm 9,5$ & $80,4 \pm 9,7$ & Aumento não significativo & $(\mathrm{p}>0,05)$ \\
\hline Sistema vestibular & $53,0 \pm 12,3$ & $64,6 \pm 9,8$ & Aumento significativo & $(\mathrm{p}<\mathbf{0 , 0 1})$ \\
\hline Índice de equilíbrio & $67,3+6,2$ & $72,5+5,7$ & Aumento significativo & $(\mathrm{p}<\mathbf{0 , 0 5})$ \\
\hline
\end{tabular}

Rine et al. ${ }^{4}$, Hirabayashi \& Iwasaki ${ }^{9}$ e Shimizu et al. ${ }^{10}$ definem os padrões de normalidade para posturografia dinâmica em crianças considerando o peso e a idade. Os dois primeiros autores estudam crianças de três anos para o estudo normativo e concluem que o sistema somatosensorial é o primeiro a chegar ao estágio de maturação, que acontece ao redor dos 3 ou 4 anos de idade. Os sistemas visuais e vestibulares apresentam maturação mais tardia.

Inúmeros são os trabalhos que versam sobre a reabilitação em adultos ${ }^{11-14}$. Este tipo de terapia também pode ser utilizado para crianças ${ }^{15}$, no entanto, muito da dificuldade em encontrar dados na literatura advém do fato de não haver uma análise quantitativa para a comprovação da melhora clínica. Por este motivo, resolvemos utilizar a PDC como recurso nesta análise.

A posturografia possibilita uma quantificação clínica precisa dos fatos observados clinicamente. A melhora clínica é facilmente percebida pelo terapeuta, pelo otorrinolaringologista e pelos familiares das crianças. A criança vestibulopata tem, na maioria das vezes, dificuldade para expressar em palavras seus sintomas e, portanto, muitas vezes a orientação da conduta e a avaliação da terapia fica muito prejudicada. No entanto, é visível sua disposição em brincar e a melhora das crises, seja em sua freqüência, seja em sua intensidade, é facilmente observada.

$\mathrm{Na}$ análise da posturografia, ressalta-se que a melhora significativa observada no equilíbrio final (índice de equilíbrio) deveu-se principalmente à melhora significativa das condições 1 e 5. Estes dados são compatíveis com os encontrados na RV em adultos ${ }^{12,13}$. A melhora da análise sensorial da função vestibular final também foi significativa, indicando ainda a melhora objetiva e mais efetiva da condição 5.
Por outro lado, outro dado relevante na avaliação foi a redução significativa na análise final da função somatosensorial, que sugere uma possível diminuição da substituição central compensatória do equilíbrio, potencializando as eferências somatosensoriais.

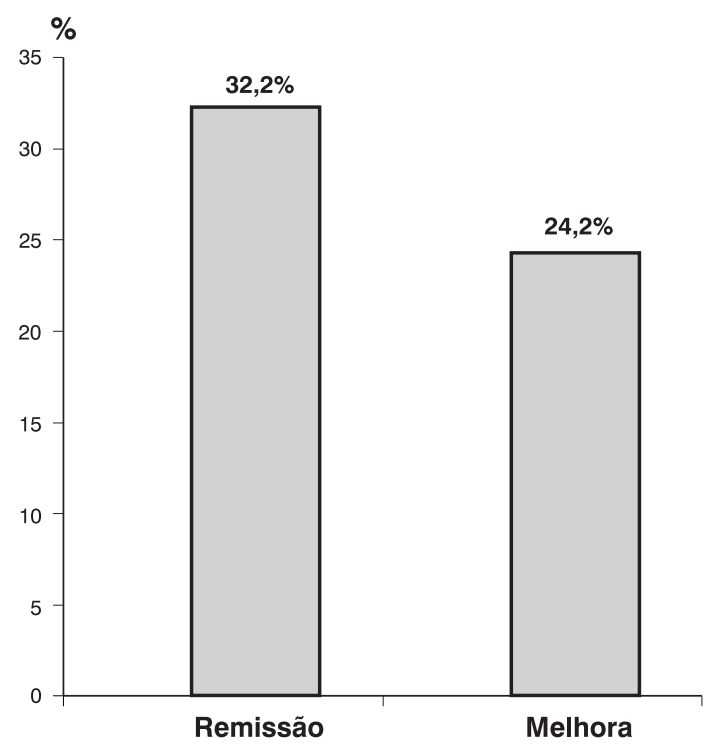

Figura 1 - Percentual de melhora do desempenho vestibular (condição 5) entre os pacientes que apresentaram remissão do quadro após o tratamento $(n=6)$ e os pacientes que apresentaram apenas melhora $(n=4)$ 
A excelente evolução clínica apresentada pelas crianças submetidas à reabilitação vestibular é semelhante àquela encontrada na literatura ${ }^{3}$, e corrobora a idéia da utilização desta terapia como método eficaz e seguro no tratamento das afecções vestibulares na faixa etária infantil.

A correlação entre a melhora clínica e a melhora à PDC mostrou-se presente em nossos pacientes. Isso nos permite sugerir que a posturografia pode auxiliar o raciocínio clínico para orientação de conduta em crianças com quadros de tontura secundária à vestibulopatia.

As crianças com cinetose tiveram um comportamento clínico diferente daquele observado nas demais. Nota-se que estes pacientes, apesar da atenuação importante deste sintoma, não ficaram inteiramente assintomáticos. Isto vem reforçar a idéia de que a avaliação através de dados clínicos qualitativos tem seus predicados que continuam sendo, portanto, fundamentais.

O estudo de um número maior de pacientes nesta faixa etária poderá trazer com mais segurança maiores subsídios técnicos, confirmando a importância da posturografia na avaliação de crianças vestibulopatas.

\section{Conclusão}

Embora a PDC não substitua a avaliação clínica convencional, agrega dados quantitativos importantes para avaliação vestibular na infância, antes e depois do tratamento instituído.

\section{Referências bibliográficas}

1. Formigoni LG, Medeiros IRT, Santoro PP, Bittar RSM, Bottino MA. Avaliação clínica das vestibulopatias na infância. Rev Bras Otorrinolaringol 1999;65(1):78-82.

2. Blayney AW, Colman BH. Dizziness in childhood. Clin Otolaryngol 1984;9:77-85.

3. Bittar RSM, Pedalini MEB, Medeiros IRT, Bottino MA, Bento RF. Reabilitação vestibular na criança: estudo preliminar. Rev Bras Otorrinolaringol 2002;68(4):496-9.

4. Fife TD, Tusa RJ, Furman JM, Zee DS, Frohman E, Baloh RW, et al. Assessment: vestibular testing techniques in adults and children: report of the Therapeutics and Technology Assessment Subcommittee of American Academy of Neurology. Neurology 2000;55(10):1431-41.
5. Hecker HC, Haug CO, Herndon JW. Treatment of the vertiginous patient using Cawthorne's vestibular exercises. Laringoscope 1974;84(11):2065-72.

6. Medeiros I, Pedalini ME, Bittar RSM, Formigoni LG. Scientific Posters: Vestibular rehabilitation in children with vestibular disorders. Otolaryngol Head Neck Surg 2001;125(2):Special Issue: S143.

7. Hubbell RN, Skoner JM. Vertigo in children. In: Fabian RL, Gluckman JL, Donald P, editores. Current Opinion in Head and Neck Surgery. Filadélfia: Lippincott, Williams \& Wilkins; 1999.p.328-34.

8. Rine RM, Rubish K, Fenney C. Measurement of sensory system effectiveness and maturational changes in postural control in young children. Pediatric Physical Therapy 1998;10:16-23.

9. Hirabayashi S, Iwasaki Y. Developmental perspective of sensory organization on postural control. Brain Dev 1995;17:111-3.

10. Shimizu K, Asai M, Takata S, Watanabe Y. The development of equilibrium function in childhood. In: Taguchi K, Igarashi M, Mori S, editores. Vestibular and Neural Front: Proceedings of the $12^{\text {th }}$ International Symposium on Posture and Gait; 3-7 Out 1994; Matsumoto, Japão. Nova Iorque: Elsevier; 1994.p.183-6

11. Cowand JL, Wrisley DM, Walker M, Strasnick B, Jacobson JT. Efficacy of vestibular rehabilitation. Otolaryngol Head Neck Surg 1998;118:49-54.

12. Mruzek M, Barin K, Nichols DS, Burnett CN, Welling DB. Effects of vestibular rehabilitation and social reinforcement on recovery following ablative vestibular surgery. Laryngoscope 1995;105:686-92.

13. Konrad HR, Tomlinson D, Stockwell CW, Norré M, Horak FB, Shepard NT, et al. Rehabilitation therapy for patients with disequilibrium and balance disorders. Otolaryngol Head Neck Surg 1992;107:105-8.

14. Gill-Body KM, Krebs DE, Parker SW, Riley PO. Physical therapy management of peripheral vestibular dysfunction: two clinical case reports. Phys Ther 1994;74(2):129-42.

15. Rine RM. Evaluation and treatment of vestibular and postural control deficits in children. In: Herdman S. Vestibular rehabilitation. $2^{\mathrm{a}}$ ed. Filadélfia: F.A. Davis Company; 2000. p.545-62.

Endereço para correspondência:

Dr. Ítalo Roberto Torres de Medeiros

Av. Dr. Arnaldo, 1927 - Sumaré

CEP 01255-000 - São Paulo, SP

E-mail: italomedeiros@uol.com.br 